\title{
CONTROL INALÁMBRICO DE UN SISTEMA LIBS PORTÁTIL BASADO EN TECNOLOGÍA BLUETOOTH
}

Iván Terceros y Omar Ormachea

\section{RESUMEN}

Se presenta el desarrollo de un sistema de control inalámbrico, basado en tecnología bluetooth, para un sistema LIBS (Laser-Induced Breakdown Spectroscopy) que utiliza un prototipo láser Nd:YAG ${ }^{++}$portátil de una potencia estimada de $10 \mathrm{MW} / \mathrm{cm}^{2}$, un espectrómetro de difracción de $0.27 \mathrm{~nm}$ de resolución y $76.9 \mathrm{~nm}$ de ancho espectral (prototipos construidos y desarrollados en el CIOE). Se optimizaron los circuitos electrónicos de control, potencia, fuente y batería del láser, obteniendo un menor tamaño y peso para el sistema LIBS. Se instalaron todos los componentes electrónicos en placas impresas generadas en una máquina de Control Numérico por Computadora TK-CNC. El sistema es capaz de funcionar tanto con una batería del tipo LiPo (Lithium Polymer) como con la red convencional de energía eléctrica. Se diseñó y construyó un primer prototipo de una pistola de medición compuesta del cabezal laser, un sistema de sincronización electrónica entre el láser y el sensor del espectrómetro (trigger) y un sistema óptico para la recolección de la luz de la muestra ablacionada por radiación. El sistema inalámbrico permite controlar al láser portátil desde un computador, haciendo posible el cambio de parámetros de funcionamiento del láser tales como la frecuencia, la energía de disparo y la toma sincronizada de datos del espectrómetro. El sistema completo que consta de un láser pulsado, un espectrómetro y el sistema de recolección de luz; tiene un peso relativamente bajo (aproximadamente $6 \mathrm{~kg}$ ) y un costo menor en un $30 \%$ de sistemas similares portátiles.

Palabras Clave: Control Electrónico Inalámbrico, Bluetooth, Espectroscopia de Emisión Atómica, LIBS, Láseres. 